\title{
State-to-state Scattering of Metastable CO Molecules from a LiF(100) Surface
}

\author{
Rienk T. Jongma, ${ }_{1}^{1}$ Giel Berden, ${ }^{1}$ Dorien van der Zande, ${ }^{1}$ Theo Rasing, ${ }^{1}$ Helmut Zacharias, ${ }^{1,2}$ and Gerard Meijer ${ }^{1}$ \\ ${ }^{1}$ Research Institute for Materials, University of Nijmegen, Toernooiveld, 6525 ED Nijmegen, The Netherlands \\ ${ }^{2}$ Physikalisches Institut, University of Münster, Wilhelm-Klemm-str. 10, 48149 Münster, Germany
}

(Received 31 May 1996; revised manuscript received 20 September 1996)

Survival of electronically excited, state-selected $\mathrm{CO}\left(a^{3} \Pi\right)$ molecules scattering from a cleaved $\mathrm{LiF}(100)$ surface is studied. Absolute survival probabilities are determined for various impact energies. Time-of-flight profiles, angular distributions, and internal state distributions of the surviving metastable $\mathrm{CO}$ molecules are measured. The dependence of their translational and rotational temperatures on the incoming velocity unambiguously indicates a direct inelastic scattering process even though the angular distributions are broad, both in plane and out of plane. [S0031-9007(97)02391-0]

PACS numbers: 82.65.Pa, 34.50.Dy, 34.50.Gb, 34.50.Lf

During the last few decades many experiments on molecular beam-surface interaction have been performed $[1,2]$, but almost exclusively with molecules in their electronic and vibrational ground states. By measuring the velocity, angular distribution, and internal state distribution of the scattered molecules for known parameters of the incoming beam and for a known scattering geometry, detailed information on the molecule-surface interaction potential is obtained [3-6].

A few groups have reported on scattering experiments in which vibrationally excited molecules are used [710]. So far, little is known about the interaction of electronically excited molecules and surfaces; the study of electronically excited molecules colliding with surfaces is thus far limited to the study of molecules residing in the different $\Omega$ components of the electronic ground state [11-13].

We here report the first state-to-state surface scattering experiment with quantum-state selected metastable molecules. By studying the electronically elastic channel only the elastic scattering properties of the process are probed; each molecule being trapped or chemisorbed will be electronically deexcited and removed from the measured signal. Apart from information obtained from timeof-flight (TOF) and angular distributions of the surviving metastable molecules, additional information on the interaction potential can be extracted from the final internal state distribution of the scattered molecules.

The experimental setup consists of a pulsed molecular beam machine connected to an ultra high vacuum (UHV) system as schematically indicated in Fig. 1. The molecular beam part consists of two differentially pumped chambers, a source chamber with a pulsed valve and a buffer chamber which contains a hexapole state selector, connected via a $0.8 \mathrm{~mm}$ diameter skimmer. A mixture of $20 \% \mathrm{CO}$ in a rare gas is used to produce the pulsed supersonic beam. Metastable CO molecules are prepared in the source chamber using direct laser excitation. The $a^{3} \Pi_{1}(v=0, J=1)$ level is populated via excitation on the $Q_{2}(1)$ line of the spin-forbidden $a^{3} \Pi \leftarrow X^{1} \Sigma^{+}$tran- sition using pulsed $206 \mathrm{~nm}$ radiation $(\sim 0.25 \mathrm{~mJ}$ in a $220 \mathrm{MHz}$ bandwidth).

The electrostatic hexapole is used to state select and focus $[2,14,15]$ the laser prepared metastable CO molecules. After passage through the hexapole, the central part of the molecular beam is blocked by a beamstop. The connection to the UHV system is via a $1.2 \mathrm{~mm}$ diameter diaphragm which is located in the "shadow" of this beamstop; only metastable $\mathrm{CO}$ molecules in the laser prepared state are bent around the beamstop and are focused through the diaphragm into the UHV system. The hexapole-beamstop-diaphragm combination transmits therefore a pure beam of metastable $\mathrm{CO}$ molecules in a single quantum state, free of ground state $\mathrm{CO}$ molecules and carrier gas atoms [15].

A cleaved $\operatorname{LiF}(100)$ crystal is mounted on a manipulator in the UHV system, $72.5 \mathrm{~cm}$ away from the excitation region. The $\mathrm{LiF}$ surface is chosen for these experiments as it is a likely candidate for which the internal energy

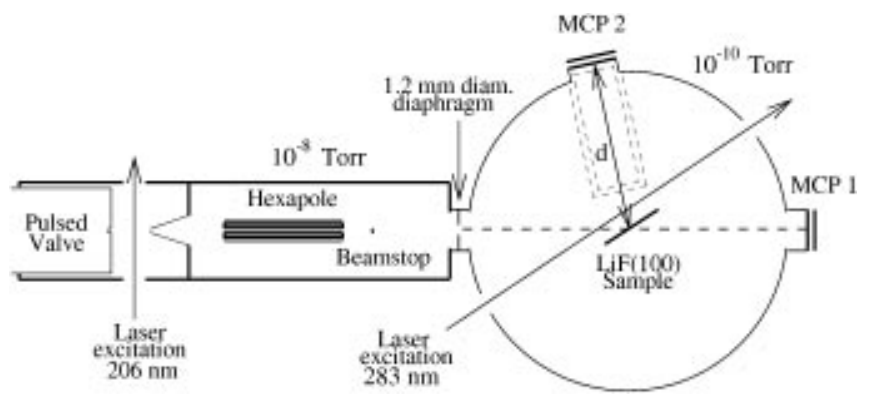

FIG. 1. Schematic view of the experimental setup. The $206 \mathrm{~nm}$ laser excitation in combination with the hexapolebeamstop-diaphragm setup allows preparation of a pure metastable $\mathrm{CO}$ beam in the UHV chamber. The TOF profile of the incoming beam is monitored with microchannel-plate (MCP) 1, while TOF profiles of scattered metastable $\mathrm{CO}$ molecules are monitored with MCP 2. Internal state distributions are probed via resonance enhanced multiphoton ionization (REMPI) with the $283 \mathrm{~nm}$ laser. Mass selective ion detection can be performed when the ion extraction assembly (dashed) is mounted in front of MCP 2. 
of the metastable $\mathrm{CO}$ molecules $(6 \mathrm{eV})$ cannot be lost during the interaction. The $\mathrm{LiF}$ samples are cleaved to provide an atomically flat surface. The flatness of the surface was checked after the experiments (in open air) with an atomic force microscope. The cleaving is done under atmospheric conditions since there appear to be no differences between air-cleaved and vacuumcleaved surfaces used in similar experiments $[10,16]$. After bakeout of the entire system, the crystal is heat cleaned in UHV at a temperature of $600 \mathrm{~K}$ for a period of $2 \mathrm{~h}$ prior to the experiments and kept at room temperature during the measurements.

Several detection schemes for metastable $\mathrm{CO}$ are implemented in the UHV chamber. Direct detection of the metastable $\mathrm{CO}$ molecules is performed with MCP detectors which allow both time- and space-resolved detection of $\mathrm{CO} a^{3} \Pi(v=0)$ [15] with an efficiency of approximately $5 \times 10^{-4}$. This detection method is not quantumstate specific and is based on Auger deexcitation. In this process the electronic energy of the metastable $\mathrm{CO}$ molecule $(6 \mathrm{eV})$ is used to create electrons at the surface of the MCP detector, which are subsequently detected. TOF profiles of the incoming metastable $\mathrm{CO}$ beam are recorded with a double staged MCP detector (Fig. 1: MCP 1) when the LiF surface is moved out of the beam. These TOF profiles directly reflect the velocity distribution of the incoming beam. TOF profiles of the scattered metastable $\mathrm{CO}$ molecules are recorded by a MCP detector at a distance $d=50 \mathrm{~cm}$ from the LiF surface (Fig. 1: MCP 2). The axis of the incoming beam and the axis of detection of the scattered molecules enclose a fixed angle of $72^{\circ}\left[\theta_{i}+\theta_{f}=72^{\circ}, \theta_{i}\left(\theta_{f}\right)\right.$ is the angle enclosed by the surface normal and incoming (scattered) beam]. Angular distributions of the scattered molecules are measured with a charge-coupled device (CCD) camera system recording the time-integrated emission of a $1^{\prime \prime}$ diameter $\mathrm{MCP} /$ phosphor screen detector mounted at a distance $d=2 \mathrm{~cm}$ from the surface.

The internal state distribution of the scattered metastable $\mathrm{CO}$ molecules is probed via one-color $(1+1)$ REMPI and mass-selective ion detection using the optional ion extraction assembly that is indicated with dashed lines in Fig. 1. The $b^{3} \Sigma^{+}(v=0) \leftarrow a^{3} \Pi(v=0)$ transition around $283 \mathrm{~nm}[17,18]$ is used as the resonant step in the REMPI scheme.

Figure 2 shows the TOF profiles of both incoming and scattered metastable CO molecules as measured for a $20 \%$ $\mathrm{CO}$ in a $\mathrm{Kr}$ mixture. In the lower trace the TOF profile of the incoming metastable $\mathrm{CO}$ molecules is shown. The time axis is rescaled to correspond to a flight path that is identical to that which the scattered metastable molecules must travel to reach MCP 2 (Fig. 1). The corresponding velocity distribution has a width (FWHM) of less than $10 \%$ of the mean velocity. The first peak in the upper trace in Fig. 2 is due to the fluorescence of metastable $\mathrm{CO}$ molecules back to the electronic ground state; the detected

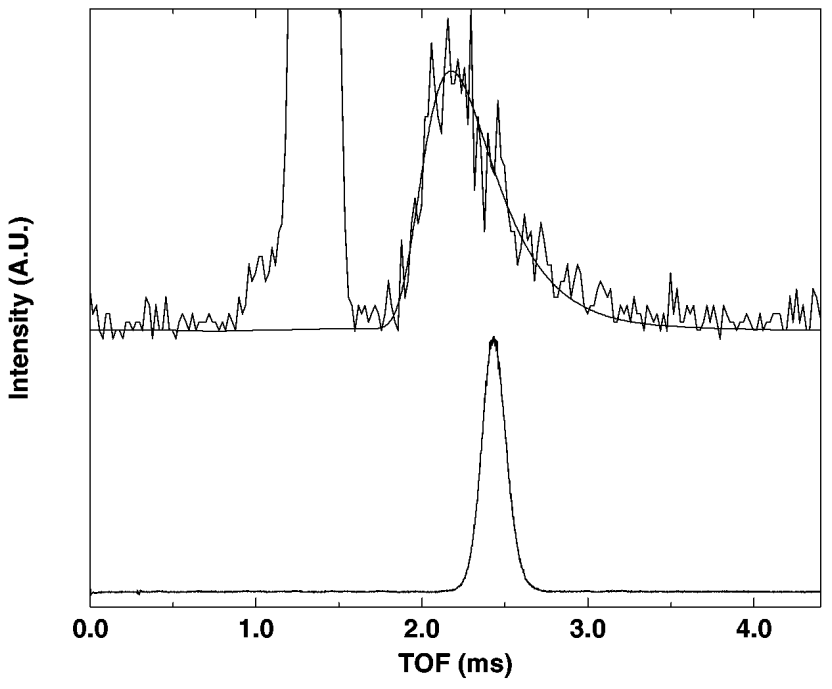

FIG. 2. Measured TOF profiles of both incoming (lower trace) and scattered (upper trace) metastable $\mathrm{CO}$ molecules for a $20 \% \mathrm{CO}$ in a $\mathrm{Kr}$ mixture. On the horizontal axis the time relative to the laser preparation is indicated. The lower trace shows the measured TOF distribution of the incoming beam (the horizontal axis is rescaled to allow direct comparison with the scattered TOF distribution). The upper trace includes the scattered TOF distribution (second peak) and the best fit. The first peak (off scale) corresponds to spontaneous fluorescence of the laser-prepared metastable molecules.

fluorescence is emitted in the direction of MCP 2 by molecules that are passing through the field of view of this detector from the moment that they enter the UHV chamber until they hit the LiF surface. The time-integrated fluorescence signal is a direct measure for the amount of incoming molecules. There is no extra fluorescence intensity visible at the time that the $\mathrm{CO}$ molecules impinge on the surface, and it is concluded that the interaction of the metastable $\mathrm{CO}$ molecules with the $\mathrm{LiF}(100)$ surface does not enhance the fluorescence significantly [19]. The second peak in the upper trace shows the TOF profile of the scattered molecules, recorded at the specular angle $\left(\theta_{i}=\theta_{f}=36^{\circ}\right)$. The time-integrated peak is a direct measure for the amount of scattered molecules. This peak can directly be compared to the TOF distribution of the incoming molecules. The TOF distribution of the scattered molecules is clearly broader, starts earlier, and has a tail to longer flight times. It can be seen from Fig. 2 that the average kinetic energy of the scattered $\mathrm{CO}$ molecules is increased after the interaction with the surface, an effect that is even more evident in TOF profiles taken for $\mathrm{CO}$ seeded in Xe. This effect is explained by the dependence of the survival probability (SP) on the velocity of the incoming and outgoing beam (vide infra). The profile of scattered CO molecules is fitted to the convolution of the measured velocity distribution of the incoming beam at the position of the LiF surface and a velocity distribution $f(v)$ given by

$$
f(v)=v^{2} e^{-m v^{2} / 2 k T_{\text {trans }}} e^{-A / v}
$$


The first two factors describe a Maxwellian velocity distribution (the MCP is a density detector, so a factor $v^{2}$ is to be used) with a Boltzmann temperature $T_{\text {trans }}$ whereas the last factor is included to take the velocity dependent SP into account. The best fitting curve with $T_{\text {trans }}=285 \mathrm{~K}$ is superimposed on the experimental trace. It should be noted that in principle the finite radiative lifetime of the scattered molecules has to be taken into account in the analysis. Neglecting this lifetime (which is at least $3 \mathrm{~ms}$ [20]), however, barely influences the determination of the Boltzmann temperature [19].

All the TOF measurements have been performed for four different carrier gasses, i.e., for four different incoming velocities. For each of the measurements the average kinetic energy of the incoming beam $\bar{E}_{\text {in }}$, the best fitting Boltzmann temperature $T_{\text {trans }}$, and the corresponding averaged kinetic energy of the outgoing beam as measured on the detector $\bar{E}_{\text {det }}$, are summarized in Table I. The best fitting Boltzmann temperature is seen to decrease gradually in going from $\mathrm{Ne}$ to $\mathrm{Xe}$. From the data listed in Table I it is seen that $\bar{E}_{\text {det }}$ is superthermal ( $>39 \mathrm{meV}$ ) for all of the carrier gases used and that for $\mathrm{CO}$ seeded in $\mathrm{Kr}$ and $\mathrm{Xe}$ the kinetic energy of the scattered molecules is higher than the averaged kinetic energy of the incoming beam. This effect can be explained by the velocity dependent SP; faster molecules have a larger probability to survive the collision with the surface as a metastable molecule than slower molecules. This leads to a velocity distribution of the detected scattered molecules that is skewed to larger velocities. The translational temperature as obtained from the fit shows that the velocity distribution of the scattered molecules is not completely accommodated to the surface.

The ratio of the integrated scattered metastable $\mathrm{CO}$ peak to the product of the integrated fluorescence peak with $\bar{v}_{\text {in }}$ is also presented in Table I for each of the carrier gases. This number is a measure for the SP. It is seen from the data given in Table I that the relative SP (normalized to 1 for CO seeded in neon) is decreasing with a decrease of the velocity of the incoming $\mathrm{CO}$ molecules. The measured velocity dependence of the SP can, in a simple model, be used to quantitatively determine the SP [19]. In this model the SP at the surface is assumed to be exponentially decaying with the interaction time with the surface, where the latter is taken to be proportional to $1 / \bar{v}_{\text {in }}+1 / \bar{v}_{\text {out }}$. Fitting the experimentally determined relative SP to this model determines the value of $A$ in Eq. (1) and yields the absolute SP for the various carrier gases as given in Table I.

For all the carrier gases the angular distribution is observed to be broad, both in plane and out of plane, and mostly resembles a cosine distribution. For a direct scattering process the parallel momentum is conserved and the angular distribution is generally close to specular [1]. In the scattering of metastable rare gas atoms from $\mathrm{LiF}(100)$ surfaces it has also been found that the angular distributions are much wider than those of the respective ground state atoms [21], which is attributed to the marked change in interaction potential. Also in our case the broad angular distributions might be explained by the detailed character of the molecule-surface interaction potential; $\mathrm{LiF}$ is an ionic crystal, and the large dipole moment of the metastable CO molecules (1.37 Debye [22]) will interact with the various $\mathrm{Li}(+)-\mathrm{F}(-)$ dipoles at the surface. Since $\mathrm{LiF}$ is a cubic crystal, these surface dipoles are both in horizontal and vertical directions, inducing not only a broad in-plane distribution but also a very broad out-ofplane distribution.

From the observed $(1+1)$-REMPI spectrum shown in Fig. 3 the internal state distribution of the scattered metastable $\mathrm{CO}$ molecules can be deduced. This spectrum is recorded for a mixture of $20 \% \mathrm{CO}$ seeded in $\mathrm{Ar}$ and an angle of incidence $\theta_{i}=51^{\circ}$. The lower trace shows the best fit to the experimental spectrum [19]. Best agreement with experiment is obtained when a single rotational temperature is used, but a nonequilibrium distribution for the three $\Omega$ components is assumed; there appears to be an overpopulation of the (initially prepared) $\Omega=1$ component with a factor of 2.5 relative to the other spin components in the final state distribution [19]. This phenomenon is observed for the other carrier gases as well. The rotational temperature as obtained from REMPI spectra measured for the various carrier gases is listed in the last column of Table I.

To conclude, in this Letter we have presented the first state-to-state scattering experiments with laser-prepared metastable molecules. The survival of electronically excited, state-selected $\mathrm{CO}$ molecules, colliding with

TABLE I. For each of the carrier gases used, the averaged kinetic energy of the incoming beam $\bar{E}_{\text {in }}$, the best fitting Boltzmann temperature $T_{\text {trans }}$, and the corresponding averaged kinetic energy of the outgoing beam as measured on the detector $\bar{E}_{\text {det }}$, the relative SP and the best fitting absolute SP (all for $\theta_{i}=36^{\circ}$ ) as well as the rotational temperature (for $\theta_{i}=51^{\circ}$ ) are given $($ surface temperature $T_{s}=298 \mathrm{~K}$ ).

\begin{tabular}{ccccccc}
\hline \hline Carrier gas & $\bar{E}_{\text {in }}(\mathrm{meV})$ & $T_{\text {trans }}(\mathrm{K})$ & $\bar{E}_{\text {det }}(\mathrm{meV})$ & Rel. SP & Abs. SP $(\%)$ & $T_{\text {rot }}(\mathrm{K})$ \\
\hline $\mathrm{Ne}$ & $113.8 \pm 1.2$ & $420 \pm 30$ & $83 \pm 5$ & 1.0 & $5.0 \pm 1.0$ & $360 \pm 30$ \\
$\mathrm{Ar}$ & $77.8 \pm 0.8$ & $340 \pm 35$ & $69 \pm 6$ & $0.68 \pm 0.11$ & $3.2 \pm 0.7$ & $293 \pm 25$ \\
$\mathrm{Kr}$ & $36.9 \pm 0.4$ & $285 \pm 50$ & $59 \pm 9$ & $0.25 \pm 0.05$ & $1.1 \pm 0.4$ & $330 \pm 25$ \\
$\mathrm{Xe}$ & $31.0 \pm 0.3$ & $260 \pm 55$ & $55 \pm 10$ & $0.20 \pm 0.06$ & $1.0 \pm 0.3$ & $243 \pm 20$ \\
\hline \hline
\end{tabular}




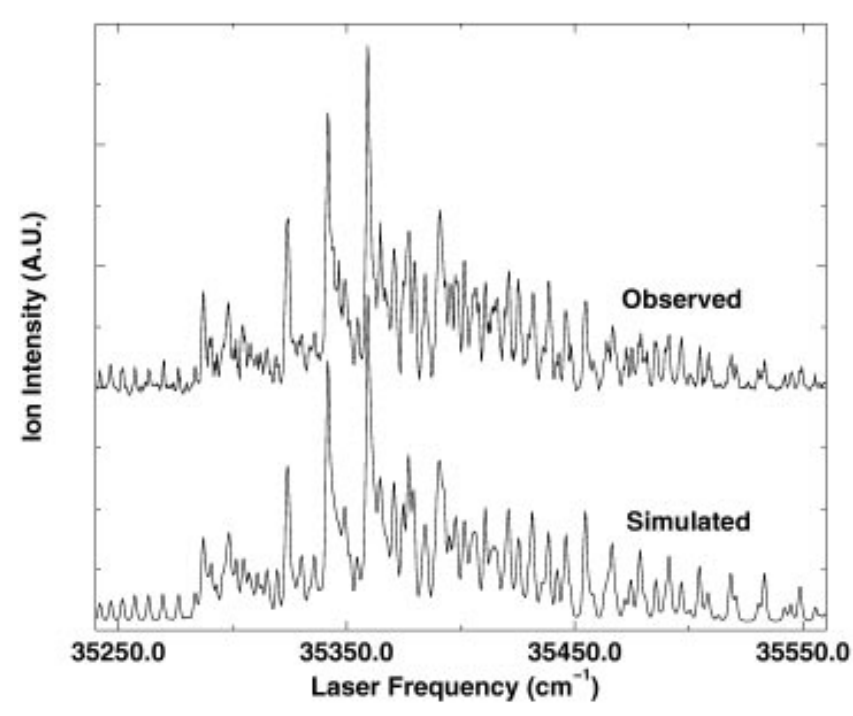

FIG. 3. Upper trace: REMPI spectrum which reflects the internal state distribution of scattered metastable $\mathrm{CO}$ molecules. The spectrum is recorded for a gas mixture of $20 \% \mathrm{CO}$ in $\mathrm{Ar}$ and $\theta_{i}=51^{\circ}$. Lower trace: Best fitting spectrum using $T_{\text {rot }}=293 \mathrm{~K}$, and an overpopulation of the $\Omega=1$ component with a factor of 2.5 relative to the other $\Omega$ components.

a cleaved $\operatorname{LiF}(100)$ surface, is analyzed using timeand space-resolved detection techniques. The absolute survival probability for metastable $\mathrm{CO}$ scattering from $\mathrm{LiF}(100)$ is observed to be between $1 \%$ and $5 \%$ for the conditions used in the experiments. The translational temperature, obtained from the TOF profiles of the surviving metastable $\mathrm{CO}$ molecules, show that the metastable $\mathrm{CO}$ molecules are scattered from the surface with not completely thermalized velocities. The rotational temperature shows a similar trend and it is concluded therefore that the metastable $\mathrm{CO}$ molecules undergo direct inelastic scattering. The angular distributions are observed to be broad which might be attributed to the strong corrugation of the electrostatic interaction potential in the $\mathrm{CO}^{*} / \mathrm{LiF}(100)$ system.

We thank Carsten Menzel for fruitful discussions and preparation of the LiF crystals, J. Gerritsen for characterizing the LiF surfaces, and C. A. Sikkens, J.G.H. Hermsen, and C.A. Timmer for technical support. The authors thank the referees for their valuable comments which improved the interpretation of the data.

[1] J. A. Barker and D. J. Auerbach, Surf. Sci. Rep. 4, 1 (1984).

[2] Atomic and Molecular Beam Methods, edited by G. Scoles (Oxford University Press, New York, 1988/ 1992), Vols. I/II.

[3] A. W. Kleyn, A. C. Luntz, and D. J. Auerbach, Phys. Rev. Lett. 47, 1169 (1981).

[4] E. W. Kuipers, M. G. Tenner, and A. W. Kleyn, Nature (London) 334, 420 (1988).

[5] D. A. Mantell, S. B. Ryali, G. L. Haller, and J. B. Fenn, J. Chem. Phys. 78, 4250 (1983).

[6] M. Asscher, W. L. Guthrie, T.-H. Lin, and G. A. Somorjai, Phys. Rev. Lett. 49, 76 (1982).

[7] J. Misewich, C. N. Plum, G. Blyholder, P. L. Houston, and R. P. Merill, J. Chem. Phys. 78, 4245 (1983).

[8] H. Zacharias, M. M. T. Loy, and P. A. Roland, Phys. Rev. Lett. 49, 1790 (1982).

[9] M. Gostein, H. Parhikhteh, and G. O. Sitz, Phys. Rev. Lett. 75, 342 (1995).

[10] J. Misewich, H. Zacharias, and M. M. T. Loy, Phys. Rev. Lett. 55, 1919 (1985).

[11] A. C. Luntz, A. W. Kleyn, and D. J. Auerbach, Phys. Rev. B 25, 4273 (1982).

[12] G. D. Kubiak, J. E. Hurst, H. G. Rennagel, G. M. McClelland, and R. N. Zare, J. Chem. Phys. 79, 5163 (1983).

[13] D. A. Mantell , Y.-F. Maa, S. B. Ryali, G. L. Haller, and J. B. Fenn, J. Chem. Phys. 78, 6338 (1983).

[14] K. H. Kramer and R. B. Bernstein, J. Chem. Phys. 42, 767 (1965).

[15] R. T. Jongma, Th. Rasing, and G. Meijer, J. Chem. Phys. 102, 1925 (1995).

[16] J. Estel, H. Hoinkes, H. Kaarmans, H. Nahr, and H. Wilsch, Surf. Sci. 54, 393 (1976).

[17] G. H. Dieke and J. W. Mauchly, Phys. Rev. 43, 12 (1933).

[18] T. Rytel, J. Mol. Spectrosc. 145, 420 (1991).

[19] R. T. Jongma, G. Berden, Th. Rasing, H. Zacharias, and G. Meijer (to be published) .

[20] T. C. James, J. Chem. Phys. 55, 4118 (1971).

[21] H. Conrad, G. Ertl, J. Küppers, W. Sesselmann, B. Woratschek, and H. Haberland, Surf. Sci. 117, 98 (1982).

[22] R. C. Stern, R. H. Gammon, M. E. Lesk, R. S. Freund, and W. A. Klemperer, J. Chem. Phys. 52, 325 (1970). 